\title{
PULMONARY HYPERTENSION AND A CONTINUOUS MURMUR DUE TO MULTIPLE PERIPHERAL STENOSES OF THE PULMONARY ARTERIES
}

\author{
BY \\ WILLIAM G. SMITH \\ From Sully Hospital (Thoracic Centre), Penarth, Glamorgan
}

(RECEIVED FOR PUBLICATION APRIL 14, 1958)

Pulmonary hypertension has been the subject of many recent studies. The varieties of pulmonary hypertension can be classified as passive, obstructive, hyperkinetic, and vasoconstrictive (Wood, 1956). In the obstructive group acute and chronic pulmonary thrombosis and embolism, chronic lung disease, collagen disease, and schistosomiasis are the usual causes.

A patient is described in whom an obstructive form of pulmonary hypertension was due to multiple bilateral stenoses of the main branches of the right and left pulmonary arteries. These congenital anomalies also gave rise to a continuous ductus-like murmur. This syndrome was first described in Sweden (Arvidsson, Karnell, and Möller, 1955) and has not been reported in Britain.

\section{CASE RePort}

A Welsh girl of 8 years was referred to Mir. Dillwyn Thomas in September, 1957. A heart murmur was first noted at the age of $6 \frac{1}{2}$ years. There was slight effort dyspnoea and one episode of faintness occurred after severe exertion. A sister, cyanosed from birth, died at home aged $3 \frac{1}{2}$ years. A brother, aged 13 years, has no abnormality on clinical examination.

The child was slightly mentally backward. The second and third toes of both feet were webbed and the fifth fingers were short and incurved, but there were no other features of mongolism. Physical development was otherwise normal. There was no cyanosis or clubbing. The hands were warm and the pulse regular and of slightly reduced volume. Blood pressure was $105 / 70 \mathrm{~mm}$. Hg. A dominant "a " wave was present in the jugular venous pulse. The heart was slightly enlarged. There were no thrills, but the right ventricle and the second sound were easily palpable at the left sternal edge. A continuous murmur with late systolic accentuation was audible at the base of the heart. At the apex the systolic component was fainter and there was no diastolic component. The continuous murmur was also widely heard over both lungs anteriorly and posteriorly. It was loudest just above and medial to the right nipple, but was almost as loud at the inferior scapular angles. The murmur clearly extended into diastcle but lacked a "machinery" quality due to the rather short diastolic component (Fig. 1). The second sound was closely split on inspiration and the pulmonary element was very loud.

Chest radiographs (Fig. 2) showed a moderate increase in heart size due to right ventricular enlargement. The pulmonary arc was full but the branches appeared normal. The aorta was left-sided.

An electrocardiogram (Fig. 3) showed a wandering pacemaker with considerable right ventricular hypertrophy and "strain," $\mathrm{T}$ wave inversion extending from $V_{1}$ to $V_{5}$.

Cardiac catheterization revealed marked pulmonary hypertension $(100 / 40 \mathrm{~mm}$. $\mathrm{Hg}$; mean, $63 \mathrm{~mm}$. $\mathrm{Hg})$ in the pulmonary trunk and proximal portions of the right and left pulmonary arteries. In the region of the main branches of both pulmonary arteries a sudden fall of pressure occurred (mean $9 \mathrm{~mm}$. $\mathrm{Hg}$ ). The catheter tip was quite free at these points and could be passed easily to the extreme periphery of both lungs where a normal wedge-pressure tracing was recorded (mean $6 \mathrm{~mm}$. $\mathrm{Hg}$ ). On withdrawing the catheter the sudden change to high pressure invariably occurred in the hilar regions well proximal to the position of wedging (Fig. 4). This phenomenon was demonstrated in all of many different branches on both sides. There was no evidence of pulmonary valvular stenosis (R.V. pressure $=98,8 \mathrm{~mm}$. $\mathrm{Hg})$ and the cardiac output was 6.5 litres per minute. Multiple blood samples showed no left-to-right shunt. The oxygen saturation in the brachial artery $(96 \%)$ and the dye-dilution curve excluded a right-to-left shunt. The dye-dilution curve recorded from a forehead oximeter after an injection of Evans blue dye (T-1824) into the pulmonary trunk showed slightly delayed appearance and build-up times. The findings were taken to indicate multiple bilateral stenoses of the main branches of the pulmcnary arteries at the approximate level of the hila. The passage of the catheter to the periphery well beyond the points of low pressure excluded inadvertent wedging and vasoconstrictive pulmonary hypertension. There was no basis for hyperkinetic pulmonary hypertension.

Tomograms showed insufficient anatomical detail, and, as it was felt that precise detail might not te 


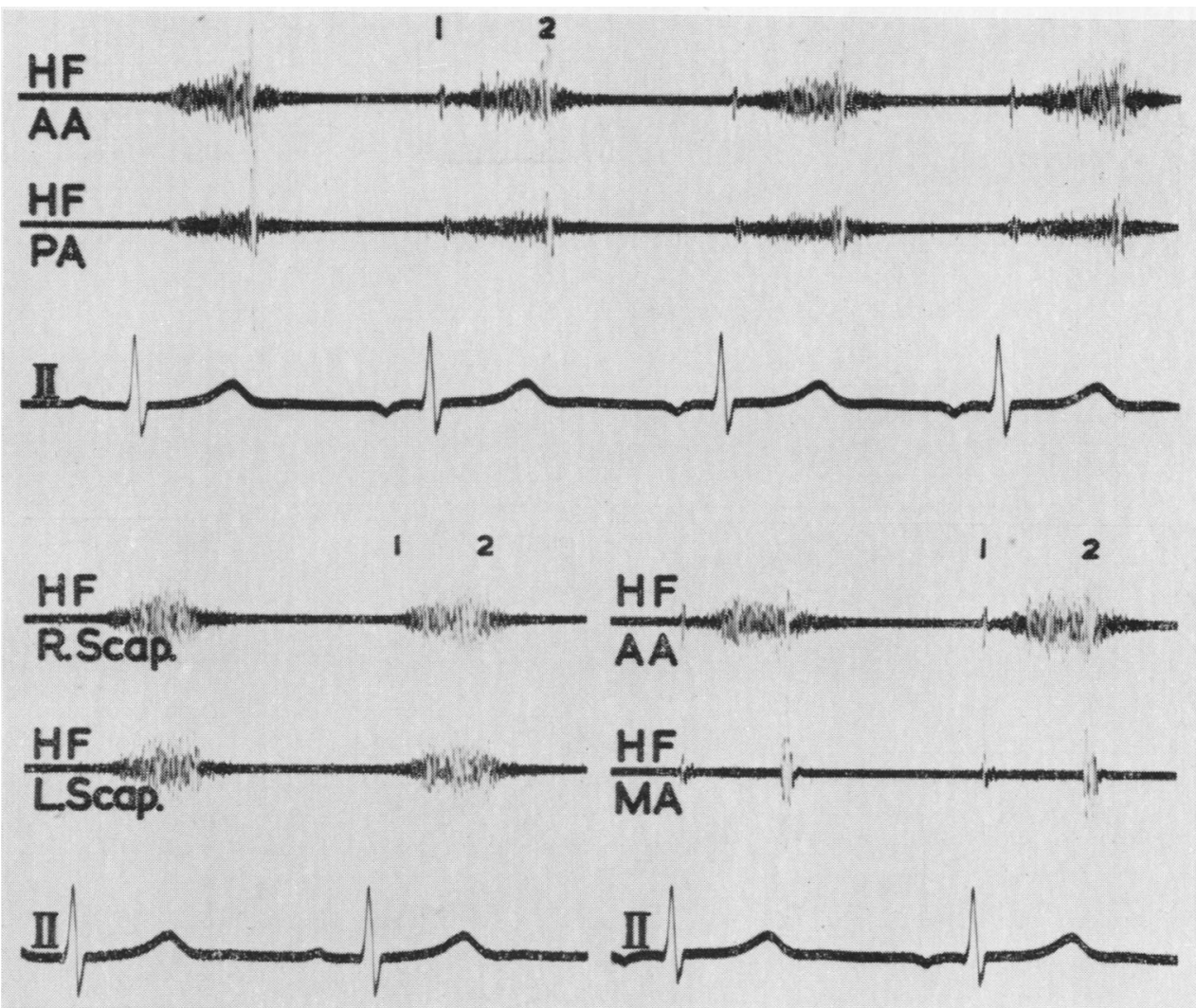

FIG. 1.-High frequency phonocardiograms showing continuous murmur and accentuation of pulmonary second sound. A.A. = aoric area, P.A.=pulmonary area, M.A.= mitral area, Scap.=inferior scapular angle.

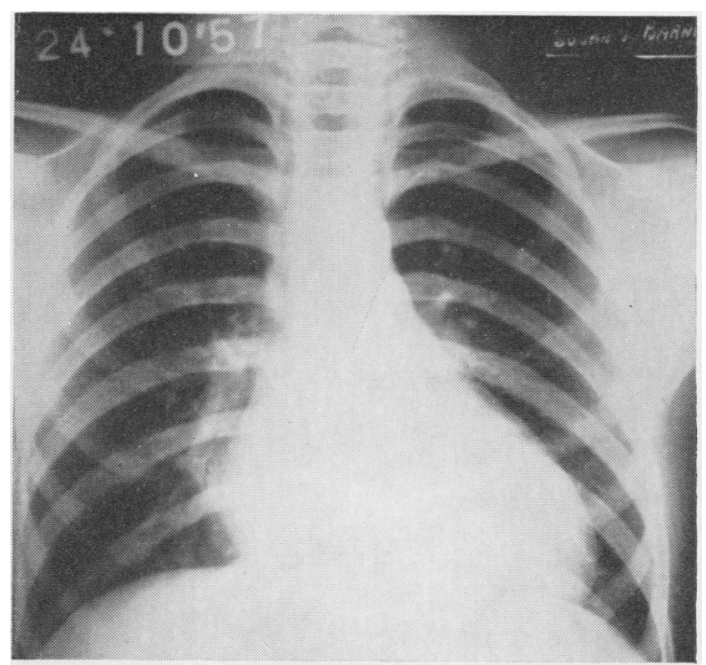

FIG. 2.-Teleradiograph showing moderate enlargement of the heart. obtained by venous angiocardiography, selective angiocardiography was performed. Under general anaesthesia a No. 9F catheter was introduced into the right saphenous vein so that the tip lay in the pulmonary trunk. Thirty millilitres of $70 \%$ sodium acetrizoate (diagonal) were injected rapidly with a manual device. Five anteroposterior films were exposed in eight seconds. On the left side, in the hilar region, multiple short and long stenoses of all the main branches were present (Fig. 5). No complete obstructions were evident. Beyond the stenoses well-marked fusiform post-stenotic dilatations were clearly seen and in one of the basal branches the dilatation extended for $5 \mathrm{~cm}$. The peripheral arborizations were normal. Unfortunately the catheter tip had been advanced a little too far and filling on the right side was unsatisfactory although one stenosis in the superior division was seen. However, the clinical and catheterization findings clearly indicated that the stenoses were bilateral. Despite the obstructions, circulation time was within normal limits as the left side of the heart and aorta were opacified five seconds after the injection. These structures were normal and there was no evidence of any shunt. 


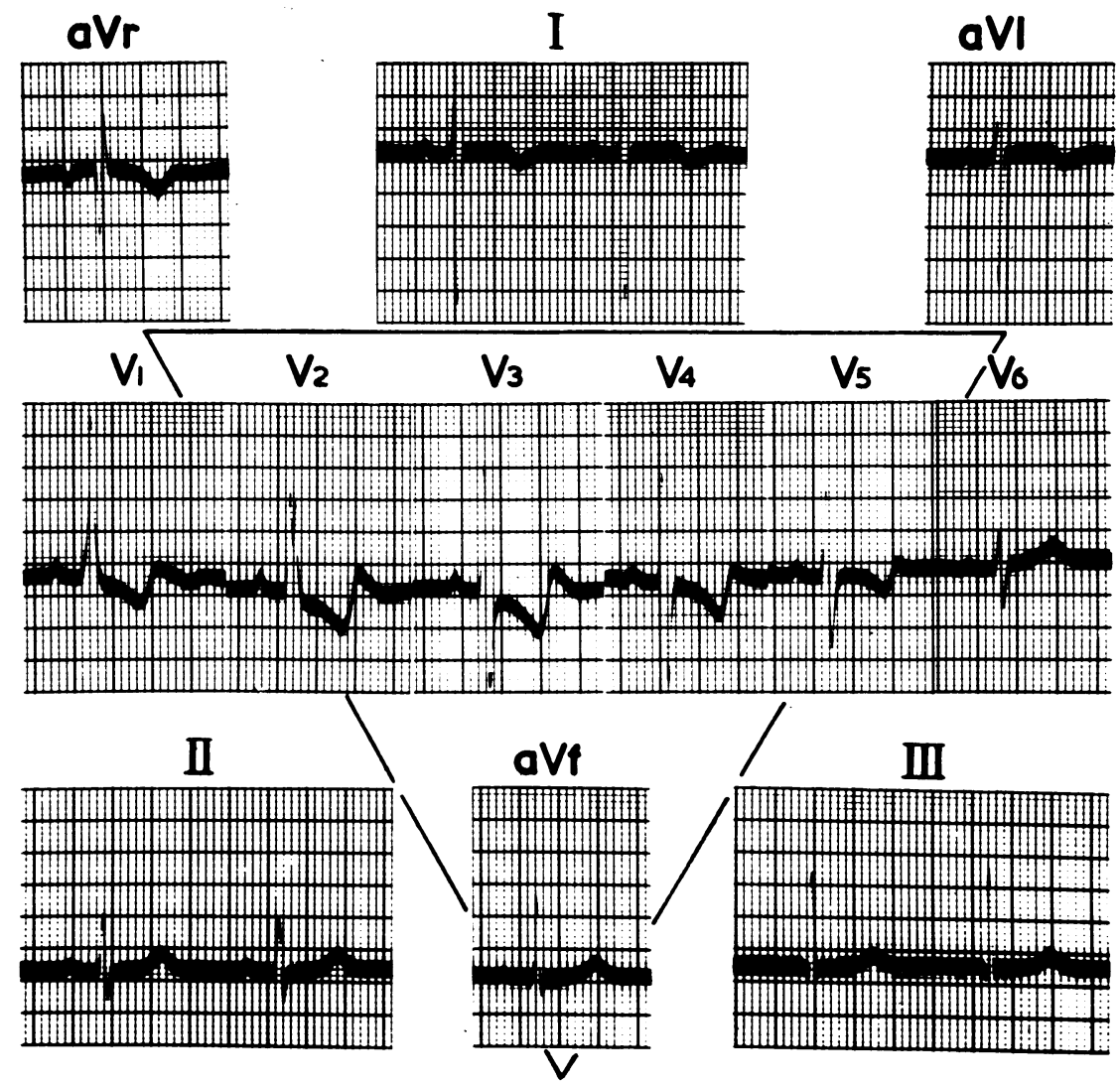

FIG. 3.-Electrocardiograph showing considerable right ventricular hypertrophy and "strain."

\section{Discussion}

In this patient the pulmonary hypertension has been caused by bilateral multiple stenoses of the pulmonary arteries. An increased resistance is exerted at the points of constriction. The poststenotic dilatations are presumably similar to those found distal to aortic and pulmonary valve stenoses and coarctation of the aorta. The continuous murmur can be attributed to blood flow through the stenoses in systole and part of diastole. Diastolic blood flow results from the high-pressure elastic reservoir of blood existing proximal to the obstructions. Study of the pressure curves obtained proximal and distal to a stenosis shows the marked pressure gradient throughout the whole of the cardiac cycle (Fig. 4). This is, of course, the haemodynamic requirement in patent ductus arteriosus and other conditions causing continuous murmurs. An analogous situation occurs in incomplete coarctation of the aorta when a continuous murmur may be heard posteriorly over the site of the coarctation.
The clinical diagnosis is not difficult if such a condition is considered. In this patient the widespread continuous murmur and the absence of central cyanosis gave a suggestive clinical clue that the murmur might be produced in the branches of the pulmonary artery. It was not a venous hum and a patent ductus arteriosus seemed unlikely. Pulmonary arteriovenous communications, which might have caused a widespread continuous murmur, should have been radiographically visible and would probably have caused central cyanosis. Pulmonary atresia with bronchopulmonary communications causing a continuous murmur ought to have caused cyanosis.

Arvidsson and others (1955) described four $\mathrm{N}_{\omega}$ patients suffering from pulmonary hypertension due to multiple stenoses of the main branches of the pulmonary arteries. Diagnosis was made by selective angiocardiography and the first case was overlooked after conventional venous angiocardiography. The findings on cardiac catheterization. other than the pulmonary hyper- 


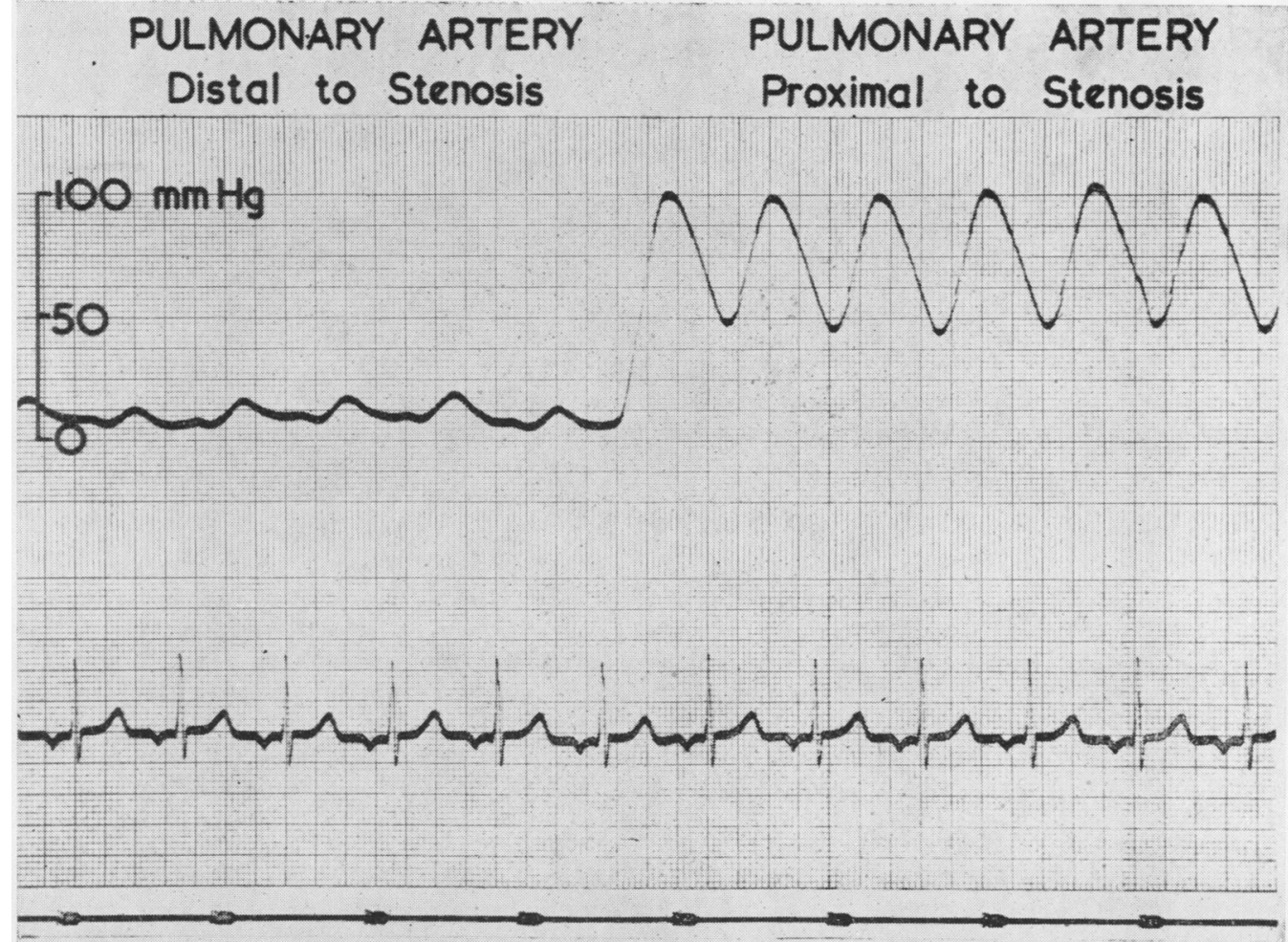

Fig. 4.-Pressure tracing obtained by withdrawal of cardiac catheter along a main branch of the right pulmonary artery towards the pulmonary trunk. The sudden pressure gradient occurred well proximal to the wedged position.

tension and blood oxygen saturations, were not given in detail. Three of the four patients were females aged 13, 15, and 33 years. The fourth was a male aged 10 years. All had pulmonary hypertension $(56 / 18,56 / 13,72 / 7,108 / 22 \mathrm{~mm}$. $\mathrm{Hg})$. The girl aged 13 years had a continuous murmur over the entire chest and the angiocardiographic appearances were almost identical with those described in this paper. The remaining patients had systolic murmurs and the angiocardiographic appearances were less striking. The woman aged 33 years had an atrial septal defect, but shunts were absent in the others. The authors believed that these were the first reported examples of pulmonary hypertension due to such anomalies. Gyllensward, Lodin, Lundberg, and Möller (1957) reported eight patients who had congenital multiple peripheral stenoses of the pulmonary arteries (two were included in the paper of Arvidsson and others, 1955). They were investigated by cardiac catheterization and selective angiocardiography. One had an annular membranous stenosis about $1 \mathrm{~cm}$. distal to the pulmonary valve which was also stenosed. This membranous stenosis was similar to that described in the monograph of Kjellberg, Mannheimer, Rudhe, and Jonsson (1955). The remaining seven patients had anatomical abnormalities similar to the present case. In two cases blind-ending vascular sacs were present in addition to the stenoses. Two of these patients were mother and son. Four patients had moderate or marked pulmonary hypertension and one patient had pulmonary valvular stenosis in addition to the peripheral stenoses. One patient had a small left-to-right shunt at atrial level. All had loud systolic murmurs usually maximal at the pulmonary area and the only patient with a continuous murmur had already been reported by Arvidsson and others (1955).

Eldridge, Selzer, and Hultgren (1957) described five patients who were shown by cardiac catheterization to have single or multiple stenoses of a major branch of the pulmonary artery. In one patient the lesions were bilateral, but in the others the constrictions were unilateral and localized. Two had continuous murmurs at the chest wall over the site of the stenosis and one underwent thoracotomy for suspected patent ductus arteriosus. All had associated congenital defects of the heart, but not 


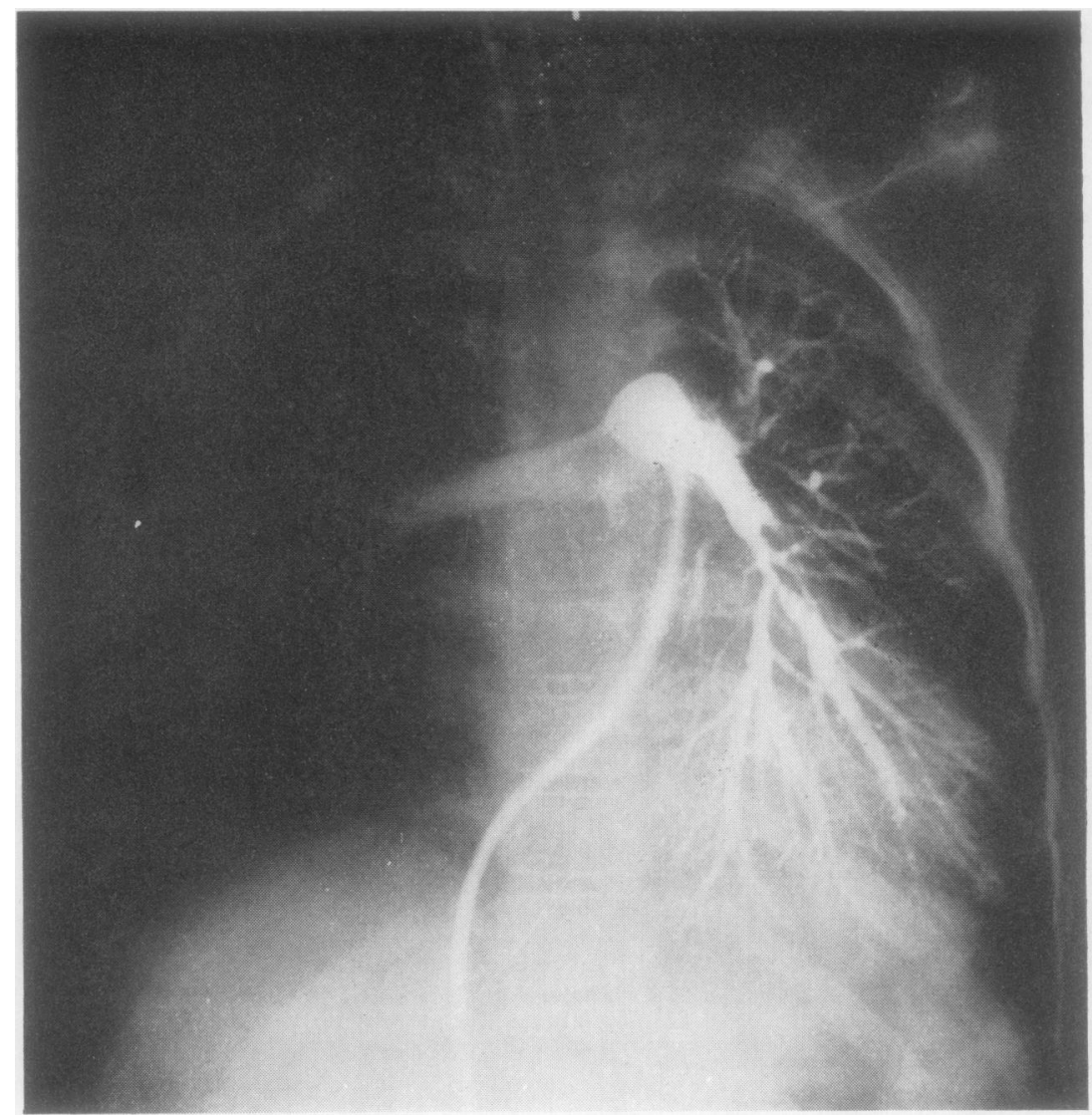

FIG. 5.-Angiocardiograph showing stenoses and post-stenotic dilatations of all main branches of the left pulmonary artery.

including pulmonary valvular stenosis. The diagnosis was made on the basis of pressure gradients occurring at one or more sites distal to the bifurcation of the pulmonary trunk. Angiocardiographic proof was not obtained, but the catheterization findings were thought to be conclusive. Two patients had a normal pressure in the pulmonary artery and only one had significant pulmonary hypertension. These workers produced continuous murmurs experimentally in the dog by partial constriction of a branch of the pulmonary artery.

Gunning (1957) reported a patient suffering from stenosis of a pulmonary artery branch to the right upper lobe. This caused a continuous murmur thought to be due to a right-sided patent ductus arteriosus. At thoracotomy the murmur was abolished by gentle pressure over the vessel in question. This patient had no left pulmonary artery, atrial and ventricular septal defects, and a right-sided aorta.
A different anatomical variety of post-valvular pulmonary artery stenosis was reported by Shumacker and Lurie (1953). The patient, who had severe congenital cyanotic heart disease, was found at thoracotomy to have marked stenosis and calcification at the bifurcation of the pulmonary trunk near the attachment of the ligamentum arteriosum. Surgical dilatation was possible and clinical improvement followed. Søndergaard (1954) described three patients with Fallot's tetralogy in whom similar abnormalities were discovered during a three-year period following his adoption of opening the pericardium to allow adequate exploration in Fallot's tetralogy. He suggested the term "coarctation of the pulmonary artery," and attributed the anomalies to obliteration of the ductus arteriosus (the Skodaic theory). Powell and Hiller (1955) described a 5-year-old boy studied by cardiac catheterization and venous angiocardiography in whom narrowings of the right and left pulmonary 
arteries at the bifurcation of the pulmonary trunk were demonstrated. A continuous murmur was audible at the pulmonary area. A similar case was reported by Coles and Walker (1956). Cardiac catheterization showed stenoses of the right and left pulmonary arteries at their origin and there was also evidence of pulmonary valvular stenosis. Williams, Lange, and Hecht (1957) reported four more patients with lesions similar to those reported by Søndergaard. These had been discovered in a five-year period amongst a total of 70 patients with congenital pulmonary stenosis. In two patients the diagnosis was made at thoracotomy and in the others by cardiac catheterization. Three patients had coexisting pulmonary valvular stenosis. The fourth patient had an accentuated pulmonary second sound as would be expected with a postvalvular stenosis. One patient had Fallot's tetralogy, one had an atrial septal defect, and a third had a ventricular septal defect.

This review of published work shows that there are three types of peripheral post-valvular pulmonary artery stenosis.

TYPE I.-Single or multiple stenoses of the pulmonary arteries. These are usually situated in the main branches in the hilar region but may be present in relatively small extrahilar branches. Post-stenotic dilatations are usually present.

TYPE II.-Stenosis of the bifurcation of the pulmonary trunk (" coarctation of the pulmonary artery").

TYPE III.-Membranous stenosis immediately distal to the pulmonary valve.

These anomalies may vary in severity and may be associated with other congenital cardiovascular malformations including pulmonary valvular stenosis. Types II and III are clinically similar to pulmonary valvular stenosis but can be differentiated by the accentuation instead of diminution and delay of the pulmonary element of the second sound. This distinction will not apply if pulmonary valvular stenosis is also present.

The writer is not qualified to discuss the embryological aspects of these anomalies, but from the practical viewpoint there are good reasons for making a distinction between the three types of lesion. If surgery is contemplated the procedure will vary according to the anomaly and an accurate diagnosis is essential. Type $\mathrm{III}$ and possibly Type IJ are amenable to surgical treatment. Despite the obvious grave long-term prognosis in the patient described in this paper, surgical treatment does not seem possible. Had the stenoses been fewer in number and more proximal in situation, resection and anastomosis as in coarctation of the aorta might have been possible.

Clinically Type I may vary in its presentation and may provide considerable problems in diagnosis. If the stenoses are multiple and severe, as in the present case, severe pulmonary hypertension and a continuous murmur may result. In slight or localized forms of the anomaly a systolic murmur without pulmonary hypertension may be the only feature. In such cases careful study of bilateral withdrawal pressure tracings obtained from the pulmonary arteries at cardiac catheterization may reveal a significant systolic pressure gradient at the site of the stenosis. A further practical point arises in connexion with the possible association of peripheral post-valvular stenoses with pulmonary valvular stenosis. The peripheral lesions may be unmasked by pulmonary valvotomy and pulmonary hypertension could result. Blount, van Elk, Balchum, and Swan (1957) reported six patients who had post-operative pulmonary hypertension in a series of 25 patients treated by open pulmonary valvotomy. They stated that no convincing explanation of this phenomenon could be provided, but it is possible that associated congenital stenoses of the peripheral branches of the pulmonary arteries might provide an explanation. These lesions could be overlooked unless specifically sought on cardiac catheterization or angiocardiography.

It seems likely that peripheral stenosis of the pulmonary arteries is not rare. An increasing awareness of its existence will lead to the uncovering of fresh cases. Cardiac catheterization may give diagnostic information if the condition is borne in mind. Precise anatomical detail can be obtained by selective angiocardiography from the pulmonary trunk or the right ventricle. If pulmonary valvular stenosis is also suspected on cardiac catheterization, selective angiocardiography should be performed from the right ventricle to allow study of the pulmonary valve.

\section{SUMMARY}

An acyanotic girl suffering from multiple bilateral peripheral stenoses of the pulmonary arteries is described. No other cardiovascular malformation was present. This congenital anomaly gave rise to a distinctive clinical syndrome of severe pulmonary hypertension and a widespread continuous murmur. The findings on cardiac catheterization were characteristic, but selective angiocardiography was necessary to demonstrate the exact site and number of stenoses.

Such a condition is probably not rare and should be considered in the investigation of otherwise 
unexplained pulmonary hypertension and in the differential diagnosis of patent ductus arteriosus and unexplained systolic murmurs. It may be overlooked by conventional venous angiocardiography. Maximum diagnostic information will be achieved by selective angiocardiography, but cardiac catheterization may be conclusive if the diagnosis is considered.

Post-valvular stenoses may occur in the peripheral branches of the pulmonary artery as in the patient described, at the bifurcation of the pulmonary trunk, or immediately beyond the pulmonary valve.

My grateful thanks are due to Mr. Dillwyn Thomas for permission to publish this case and for his encouragement and advice; to Dr. Paul Wood for helpful advice; to Mr. and Mrs. Gwilym Jones for the photographs and to Miss Enid Jackson for secretarial assistance. I am particularly grateful to Dr. L. R. West for valuable help with several aspects of the preparation of this paper.

\section{REFERENCES}

Arvidsson, H., Karnell, J., and Möller, T. (1955). Acta radiol. (Stockh.), 44, 209.

Blount, S. G., Elk, J. van, Balchum, O. J., and Swan, H. (1957) Circulation, 15, 814

Coles, J. E., and Walker, W. J. (1956). Amer. Heart J., 52, 469.

Eldridge, F., Selzer, A., and Hultgren, H. (1957). Circulation, 15, 865.

Gunning (1957). Thorax, 12, 34.
Gyllensward, A., Lodin, H., Lundberg, A., and Möller, T. (1957) Pediatrics, 19, 399.

Kjellberg, S. R., Mannheimer, E., Rudhe, U., and Jonsson, B. (1955). Diagnosis of Congenital Heart Disease, p. 115. The Year Book Publishers, Chicago.

Powell, M. L., and Hiller, H. G. (1955). Med. J. Aust., 1, 272.

Shumacker, H. B., and Lurie, P. R. (1953). J. thorac. Surg., 25, 173.

Sendorgaard, T. (1954). Dan. med. Bull., $1,46$.

Williams, C. B., Lange, R. L., and Hecht, H. H. (1957). Circulation, 16, 195 .

Wood, P. (1956). Diseases of the Heart and Circulation, 2 nd ed Eyre and Spottiswoode, London.

\section{ADDENDUM}

Since the above paper was submitted a 6-yearold boy has been found to have a stenosis of the right pulmonary artery at the bifurcation of the pulmonary trunk, slight pulmonary hypertension, and an atrial septal defect. A continuous murmur with a short diastolic component was maximal below the right clavicle. 